\title{
Proximal Renal Tubular Acidosis
}

National Cancer Institute

\section{Source}

National Cancer Institute. Proximal Renal Tubular Acidosis. NCI Thesaurus. Code

C123218.

Impairment in renal proximal tubule bicarbonate reabsorption that results in a

hypokalemic hyperchloremic metabolic acidosis, which is most commonly associated with renal Fanconi syndrome. 